\title{
Vascular Thrombosis Response
}

National Cancer Institute

\section{Source}

National Cancer Institute. Vascular Thrombosis Response. NCI Thesaurus. Code C147909.

Guidelines for monitoring and reporting vascular thrombosis response. These guidelines are consistent with RECIST1.1 and consider thrombosis as a non-measurable disease. 